\title{
Fractional Statistics in Three Dimensions: Compact Maxwell-Higgs System
}

\author{
H. Fort and R. Gambini \\ Instituto de Física, Facultad de Ciencias, Universidad de la \\ República. Tristán Narvaja 1674, 11200 Montevideo, Uruguay.
}

March 15, 2018

\begin{abstract}
We show that a $(3+1)$-dimensional system composed of an open magnetic vortex and an electrical point charge exhibits the phenomenon of Fermi-Bose transmutation. In order to provide the physical realization of this system we focus on the lattice compact scalar electrodynamics $S Q E D_{c}$ whose topological excitations are open Nielsen-Olesen strings with magnetic monopoles attached at their ends.
\end{abstract}


Some years ago it was showed that a system composed of a Nambu charged closed string interacting with a Kalb-Ramond [1] point source display fractional statistics [2]. In a previous paper [3] we addressed the issue of supplying the physical realization of this, in principle, ad hoc system. We found that a closed NielsenOlesen $(\mathrm{N}-\mathrm{O})$ string vortex [4] in presence of an ordinary electric charge exhibits the phenomenon of Fermi-Bose transmutation. The $\mathrm{N}-\mathrm{O}$ vortices are solitons of the Maxwell-Higgs theory or the scalar electrodynamics $(S Q E D)$, the relativistic generalization of Ginzburg-Landau theory of superconductivity, analogous to the Abrikosov vortices in a type II superconductor. It turns out that the path integral of the Maxwell-Higgs system can be expressed in terms of the $N$ - $O$ string variables [5]. In this string representation a nontrivial long-range topological interaction of $\mathrm{N}-\mathrm{O}$ strings with particles of electric charge $Q e$ appears whenever $Q e$ is not an integer multiple of $N e$, the charge of the Higgs field. This is a four-dimensional analogue [6] of the Aharonov-Bhom effect. This long-range interaction involves the four-dimensional analogue of the Gauss linking number for loops in three dimensions i.e. the linking number $\ell k\left(\sigma, j_{C}\right)$ of world sheets of the strings and the current $j_{C}$ of the charge $Q e$. The effect of this linking number contribution is to turn the bosonic propagator into a spinorial propagator.

In this letter we consider the extension of the previous results to the case of the compact version of the Maxwell-Higgs field theory on the lattice, the $S Q E D_{c}$. Unlike the ordinary (non-compact) $S Q E D$, the path integral of this model can be expressed in terms of $\mathrm{N}$-O string vortices with magnetic monopoles at their ends (see below). One motivation to consider this extension relies on the fact that this model contains the main ingredients to reflect somehow the physics of a real system. We have in mind a 'C-shaped' ferromagnet with a slab of a type-II superconductor inbetween. In fact, quantum-mechanically, a charge always sees 'solenoids' irrespective of the nature of magnetic sources [7]. In other words, a charge would take a magnet for an equivalent collection of thin solenoids or Dirac strings. Then, provided a pair of monopole-antimonopole embodied in the $S Q E D_{c}$ lattice model are fixed, it is reasonable to regard them as the poles of the ferromagnet. The magnetic flux get trapped inside the superconductor within the $\mathrm{N}$-O strings which connect the magnetic poles which are the equivalent of the the Abrikosov magnetic flux tubes of the type II superconductor. The external charge $Q e$ can be regarded as a fixed doping.

The $S Q E D_{c}$ describes the interaction of a compact gauge field $\theta_{\mu} \in(-\pi, \pi]$ with a scalar field $\phi=|\phi| e^{i \varphi}$ with charge $N e$. The self-interaction of the scalar field is given by the potential $V=\lambda\left(|\phi|^{2}-a^{2}\right)^{2}$. For simplicitly, we consider the limit 
$\lambda \rightarrow \infty$ which freezes the radial degree of freedom of the Higgs field. This is not a strong restriction, in fact it is known that the numerical results already obtained at $\lambda=1$ are indistinguishable from the frozen case [8]. Thus the dynamical variable is compact: $\varphi \in(-\pi, \pi]$.

Theories with Abelian compact variables contain topological excitations: vortices in the $X Y$ model, monopoles in the pure compact electrodynamics, $N$ - $O$ strings in the non-compact scalar electrodynamics, etc. There is one type of topological object for each compact variable. The $S Q E D_{c}$ model we are considering has two compact variables and therefore two types of topological excitations: $N-O$ vortices and magnetic monopoles. This model is known to possess three phases namely, confining, Coulomb and Higgs [8]. The Higgs phase is the only that supports both magnetic vortices and monopoles.

There are several examples in the lattice field theory such that a change of variables in the partition function allows for a formulation of the theory in terms of the physical excitations. Banks, Kogut and Myerson [9] introduced a general tranformation which gives rise to a description of any abelian gauge theory with compact variables in terms of variables on the dual lattice associated with its corresponding topological excitations. This was a generalization of the technique used by Jose, Kadanoff, Kirkpatrick and Nelson [10] to derive the Kosterlitz-Thouless [11] phase transition in terms of vortices for the $D=2 X Y$ model. Given a $D$ dimensional lattice theory with abelian compact variables on $c_{k-1}$ cells $(k=1$ : sites and spin theory, $k=2$ : links and gauge theory, $k>2$ hypergauge theory), by means of the Banks-Kogut-Myerson transformation, one arrives to the topological expression of the partition function given by

$$
Z_{T} \propto \sum_{{ }^{*} t_{i}} \prod_{i} \exp \left[-2 \pi^{2} \alpha_{i} \sum_{c_{D-k-1}}{ }^{*} t_{i} \hat{\Delta}_{i}{ }^{*} t_{i}\right]
$$

where the index $i$ number the topological excitations, $\alpha_{i}$ and $\hat{\Delta}$ are their respective coupling constant and propagator and ${ }^{*} t_{i}$ denotes an integer variable attached to the $c_{D-k-1}$ cells of the dual lattice which correspond to the world "trajectories" of the $D-k-2$ dimensional topological excitations. In four dimensions, one has world sheets for $k=1$ and world trajectories for $k=2$.

In order to express the path integral of $S Q E D_{c}$ in terms of its topological excitations we consider the partition function for the Villain [12] form of the lattice 
action 1 which is given by

$$
\begin{array}{r}
Z=\int_{-\pi}^{\pi}(D \theta) \int_{\pi}^{\pi}(D \varphi) \sum_{n} \sum_{l} \\
\exp \left[-\sum_{c_{2}} \frac{\beta}{2}(d \theta+2 \pi n)^{2}-\sum_{c_{1}} \frac{\kappa}{2}(d \varphi+2 \pi l+N \theta)^{2}\right],
\end{array}
$$

where $D \theta(D \varphi)$ denotes the integral over all link $c_{1}$ (site $c_{0}$ ) variables $\theta(\varphi), \beta=\frac{1}{e^{2}}$ is the gauge coupling constant, $\kappa$ is the Higgs coupling constant and $n_{c_{2}}\left(l_{c_{1}}\right)$ are integer variables defined at the lattice plaquettes $c_{2}$ (links $\left.c_{1}\right)$. Fixing the gauge $d \varphi=0$ and parametrizing the $n$ variables as

$$
n=n[m]+d q
$$

where $n[m]$ is a solution of

$$
d n[m]=m,
$$

we can extend the compact variable $\theta \in(-\pi, \pi)$ to a non-compact $A=\theta+2 \pi q$ $\in(-\infty,+\infty)$. Performing the Gaussian integration and some algebra we get:

$$
Z=\sum_{n} \sum_{l} \exp \left[-2 \pi^{2} \beta\left(d n, \frac{1}{\square+M^{2}} d n\right)-2 \pi^{2} \kappa\left(N n+d l, \frac{1}{\square+M^{2}} N n+d l\right),\right.
$$

where $M^{2}=N \frac{\kappa}{\beta}$ is the mass acquired by the gauge field due to the Higgs mechanism, and $(. . . .)=.\sum_{c_{k}}$. From (3) and (四):

$$
\begin{array}{r}
m=d n, \\
\text { and taking } \\
\sigma=N n+d l,
\end{array}
$$

by performing a duality transformation we arrive to the topological representation of the path integral

$$
\begin{array}{r}
Z_{T}=\sum_{{ }^{*} m} \sum_{{ }^{*} \sigma} \exp \left[-2 \pi^{2} \beta\left({ }^{*} m, \frac{1}{\square+M^{2}}{ }^{*} m\right)\right. \\
\left.-2 \pi^{2} \kappa\left({ }^{*} \sigma, \frac{1}{\square+M^{2}}{ }^{*} \sigma\right)\right],
\end{array}
$$

${ }^{1}$ We choose the Villain form instead of the ordinary Wilson form only for simplicity, with the Wilson action it is possible to repeat all that we do here. 
where the ${ }^{*}$ denotes the Hodge dual, the ${ }^{*} m$ are integer closed 1 -forms $\left(\partial^{*} m=\right.$ $\partial \partial n \equiv 0)$ on the dual lattice, which correspond to magnetic monopole loops, and the ${ }^{*} \sigma$ are integer two-forms on the dual lattice whose border are the monopole loops i.e. from (6) and (17) we have

$$
\begin{aligned}
\partial^{*} \sigma=\partial\left(N^{*} n+\partial^{*} l\right) & =N \partial^{*} n \\
& =N^{*} m .
\end{aligned}
$$

Let us focus our attention for a moment on the meaning of equation (9). For example, in a type II superconductor $N=2$ (the charge of the condensate of Cooper pairs is $2 e$ ) then the magnetic flux is quantized as $\Phi_{0}=\frac{2 \pi}{2 e}$ ? therefore the orbital angular momentum of an electron around a unit vortex is $l_{z}=\frac{1}{2}-e \frac{\Phi_{0}}{2 \pi}=0$ and the composite is a boson 14]. On the other hand, the magnetic flux carried by a Dirac string is an integer multiple of $\frac{2 \pi}{e}$. So, equation (9) shows that the magnetic field of a pair monopole-antimonopole (the ends of a Dirac string ) is squeezed in two $\mathrm{N}-\mathrm{O}$ string vortices.

Thus, we have arrived to an expression of the path integral as a sum over surfaces ${ }^{*} \sigma$ on the dual lattice which are the world sheets of string vortices (closed and open with magnetic monopoles at their ends). These strings are obtained by intersecting the closed world sheets with a plane $t=$ constant. The quantum average of the Wilson loop for the charge $Q e, W_{Q}(C)=\exp \left[i Q\left(A, j_{C}\right)\right]$, can be written in the topological representation, repeating the same steps which lead from eq.(2) to eq.(8), as

$$
\begin{aligned}
<W_{Q}(C)>_{T}=\frac{1}{Z_{T}} \sum_{{ }^{*} m} \sum_{{ }^{*} \sigma} \exp [ & -2 \pi^{2} \beta\left({ }^{*} m, \frac{1}{\square+M^{2}}{ }^{*} m\right) \\
& -2 \pi^{2} \kappa\left({ }^{*} \sigma, \frac{1}{\square+M^{2}}{ }^{*} \sigma\right) \\
& -\frac{Q^{2} e^{2}}{2}\left(j_{C}, \frac{1}{\square+M^{2}} j_{C}\right) \\
+ & \left.2 \pi i \frac{Q}{N}\left(j_{C}, \frac{1}{\square+M^{2}} \partial \sigma\right)\right] .
\end{aligned}
$$

Using that $\partial j_{C}=0$ we have that

$$
\left(j_{C}, \frac{1}{\square+M^{2}} \partial \sigma\right)=\left(j_{C}, l\right)-M^{2}\left(j_{C}, \square^{-1} l\right),
$$

and then we get

\footnotetext{
${ }^{2}$ The flux in high $T_{c}$ superconductors like the $\mathrm{YBa}_{2} \mathrm{Cu}_{3} \mathrm{O}_{7}$ is also quantized in units of the magnetic flux quantum $\Phi_{0}=\frac{2 \pi}{2 e}[13]$.
} 


$$
\begin{array}{r}
<W_{Q}(C)>_{T}=\sum_{{ }^{*} n} \sum_{{ }^{*} l} \exp \left[-2 \pi^{2} \beta\left({ }^{*} m, \frac{1}{\square+M^{2}}{ }^{*} m\right)\right. \\
-2 \pi^{2} \kappa\left({ }^{*} \sigma, \frac{1}{\square+M^{2}}{ }^{*} \sigma\right) \\
-\frac{Q^{2} e^{2}}{2}\left(j_{C}, \frac{1}{\square+M^{2}} j_{C}\right) \\
-2 \pi i Q \frac{\kappa}{\beta}\left(j_{C}, \frac{1}{\square+M^{2}} l\right) \\
\left.+2 \pi i \frac{Q}{N} \ell k\left(\sigma_{0}, j_{C}\right)\right] .
\end{array}
$$

The first four terms in the exponent describe short range (Yukawa) interactions. The last long-range term is the linking number $\ell k\left(\sigma_{0}, j_{C}\right)$ of the the current $j_{C}$ generated by the charge $Q e$ and the world-sheets ${ }^{*} \sigma_{0}={ }^{*} \sigma-N^{*} n$ which are closed surfaces formed by two open pices: the world-sheets of the $\mathrm{N}-\mathrm{O}$ and the world-sheets of the Dirac strings. The explicit expression of $\ell k$ is

$$
\ell k=\left({ }^{*} j_{C},{ }^{*} l\right)=\left({ }^{*} j_{C}, \frac{1}{\square} d^{*}(\sigma-N n)\right) .
$$

Therefore, if we neglect the Yukawa short-range interaction terms of the Wilson loop average (13) we get an expression that has exactly the form obtained in Ref. [15] from the $(3+1)$ generalization of the $(2+1)$ Polyakov's 16 construction which included a bosonic-string in electromagnetic interaction described by a potential $A_{\mu}$ and under the action of a Kalb-Ramond antisymmetric background field $B_{\mu \nu}$. These fields are coupled through a four-dimensional analogous to the Chern-Simons interaction:

$$
\int B \wedge F
$$

where $F$ is the two-form electromagnetic field strength. The path integral (in the continuoum) of this system may be cast in the form:

$$
Z=\sum_{\sigma} e^{-T S(\sigma)} \exp \left[-\frac{i}{4 \pi^{2}} \oint_{C} d x \int_{\sigma_{C}} d \sigma_{C}\left(x^{\prime}\right)^{*}\left(d \frac{1}{\left|x-x^{\prime}\right|^{2}}\right)\right] .
$$

As in Ref.[16], the integral in the exponential is a topological number: the selflinking number $\ell k\left(\sigma_{C}, C\right)$ which measures the number of times the closed path $C$ links the sheet $\sigma_{C}$ with border $C$ in four dimensions. Thus there is a difference 
between the expression we have obtained here, involving a linking number, and that of (16). In fact, the four-dimensional extension of Polyakov's construction leads to a singular expression of the self-linking number, and some regularization procedure or framing is needed. The regularized self-linking number is equal to the sum of the twist of the framed loops plus the writhe of the original loop. Polyakov neglects in the regularization procedure the contribution of the writhe and he only considers the twist contribution of the linking number. A ratianale for this choice is that the writhe contribution is related with orbital effects and not with intrinsic angular momentum. Here, we proceed with the same philosophy. An analogous situation seems to arise in when one studies the transmutation phenomena following the Wilczek approach [17] and consider the 'return' flux which closes the vortex line [18]. Then, one has two vortices with opposite magnetic field and both contribution to the angular momentum of the composite cancel out.

Now, one can prove the transmutation, whenever $\frac{Q}{N} \neq$ integer, by following the same steps of [15]. The main idea is that the action appearing in (16) leads after a Dirac quantization to a set of variables that behave as Pauli matrices and reproduce the propagator of the fermionic string.

One could have followed a similar approach in the $(2+1)$ case. Starting from the charge vortex pair, one could have studied the transition amplitude by computing the Feynman path integral of a charged particle in the presence of a magnetic vortex. This computation would have led to the ordinary Gauss linking number and to the action already considered in Ref. [16].

Sumarizing, we have proved that the open string-like excitations of the MaxwellHiggs system in presence of an external charge undergo Fermi-Bose transmutation. The possibility to connect this result with a real system relies on the fact that, from the quantum-mechanical point of view, a charge interacts with a magnetic source only through the vector potential. Thus, a charge always sees a magnet as an equivalent collection of thin solenoids or Dirac strings. The fact that in this case the topological contribution arises from a linking number instead of a self-linking number seems to be irrelevant due to the fact that the vortices in a type II superconductor, even in the high $T_{c}$ superconductors [19], only have small displacements and therefore one can consider that the world-line of the (fixed) charge remains close to the vortex world-sheet. However, it is still not completely clear if the transmutation here observed could affect the vortex dynamics and the behaviour of a superconductor. 


\section{References}

[1] M. Kalb and P. Ramond, Phys. Rev. D9 (1974) 2273.

[2] R. Gambini and L. Setaro, Phys. Rev. Lett. 65 (1990) 2623.

[3] H. Fort and R. Gambini, Preprint IFFC-95-01, Feb. 1995, hep-th/9502008.

[4] H.B.Nielsen and P.Olesen, Nucl. Phys. B61 (1973) 45.

[5] E. T. Akhmedov, M. N. Chernodub, M.I.Polikarpov, and M.A.Zubkov, Preprint ITEP-95-24, June 1995, hep-th/9505070.

[6] M. G. Alford and F. Wilczek, Phys. Rev. Lett. 62 (1989) 1071.

[7] J. Maeda and K. Shizuya, Z. Phys. C60 (1993) 265.

[8] K. Jansen, J. Jersak, C.B. Lang, T. Neuhaus and G. Vones, Nucl. Phys. B265 (1986) 129.

[9] T. Banks, R. Myerson and J.B. Kogut, Nucl. Phys. B129 (1977) 493.

[10] J. Jose, L-P. Kadanoff, S. Kirkpatrick and D.R. Nelson, Phys. Rev.B16 (1977) 1217.

[11] J. Kosterlitz and D. Thouless, J.Phys. C6 (1973) 1181.

[12] J. Villain, J. Phys. (Paris) 36 (1975) 581.

[13] P. L. Gammel, D. J. Bishop, G. J. Dolan, J. R. Kwo, C. A. Murray, L. F. Schneemeter and J. V. Waszczak, Phys. Rev. Lett. 59 (1987) 2592.

[14] F. Wilczek, Phys. Rev. Lett. 48 (1982) 1144.

[15] X. Fustero, R. Gambini and A. Trias, Phys. Rev. Lett. 62 (1989) 1964.

[16] A. M. Polyakov, Mod. Phys. Lett. A3 (1988) 325.

[17] F. Wilczek, Phys. Rev. Lett. 49 (1982) 957.

[18] M. Peshkin, Phys. Rep. 80 (1989) 376.

[19] Z. Yao, S. Yoon, H. Dai, S. Fan and C. M. Lieber, Nature 371 (1994) 777. 